\title{
Significance of malondialdehyde, superoxide dismutase and endotoxin levels in Budd-Chiari syndrome in patients and a rat model
}

\author{
DE-LEI CHENG ${ }^{1,2^{*}}$, NAN ZHU ${ }^{3 *}$, CHENG-LI LI $^{1}$, WEI-FU LV ${ }^{2}$, WEI-WEI FANG ${ }^{2}$, \\ YA LIU $^{4}$ and CHUAN-TING LI ${ }^{1}$
}

\begin{abstract}
${ }^{1}$ Department of Magnetic Resonance Imaging, Shandong Medical Imaging Research Institute, Shandong University, Jinan, Shandong 250021; ${ }^{2}$ Department of Interventional Radiology, Anhui Provincial Hospital, Hefei, Anhui 230001;

${ }^{3}$ Department of Radiology, People's Hospital of Wuhe County, Bengbu, Anhui 233300; ${ }^{4}$ Department of

Interventional Radiology, The Third People's Hospital of Hefei, Hefei, Anhui 230022, P.R. China
\end{abstract}

Received March 27, 2018; Accepted October 2, 2018

DOI: $10.3892 /$ etm.2018.6835

\begin{abstract}
Budd-Chiari syndrome (BCS) is a rare clinical syndrome caused by the obstruction of hepatic venous outflow. In theory, hepatic congestion and hypoxia induce pathological damage and changes in the liver. However, at present, laboratory evidence supporting this theory is lacking. The aim of the present study was to assess the expression and significance of the hypoxia-associated indicators malondialdehyde (MDA), superoxide dismutase (SOD) and endotoxin (ET) in the liver and serum of subjects with BCS. An animal model of BCS was established by partial ligation of the inferior vena cava (IVC) in rats. The levels of MDA, SOD and ET in the serum of BCS patients, as well as in the liver and serum of rats with BCS, were detected and analyzed. In human patients with BCS, the serum levels of MDA, ET and SOD were significantly different from those in healthy control subjects. In the animal model, similar trends were observed regarding the MDA, ET and SOD levels in liver homogenate and serum $(\mathrm{P}<0.05)$, the degree of which was more pronounced in the liver homogenate than in the serum. At 6 weeks after the surgery, these indicators reached peak/valley levels in the experimental group and were at least partially restored by week 12 . A negative correlation between MDA and SOD, a positive correlation between MDA and ET, and a negative correlation between
\end{abstract}

Correspondence to: Professor Chuan-Ting Li, Department of Magnetic Resonance Imaging, Shandong Medical Imaging Research Institute, Shandong University, 324 Jing Five Road, Jinan, Shandong 250021, P.R. China

E-mail: lichuanting123@yeah.net

${ }^{*}$ Contributed equally

Key words: Budd-Chiari syndrome, hypoxia, reactive oxygen species, endotoxin
SOD and ET was identified. In conclusion, the levels of hypoxia-associated indicators significantly changed with $\mathrm{BCS}$ progression, suggesting that hypoxia is a major factor in the pathogenesis of BCS.

\section{Introduction}

Budd-Chiari syndrome (BCS) is a serious clinical syndrome caused by the obstruction of hepatic venous outflow from the hepatic venules to the atriocaval junction (1-3). Without any active and effective treatment, the symptoms themselves are not relieved and are progressively aggravated. The natural prognosis of patients with BCS is poor. Patients with BCS may die from liver failure in the acute phase. In the chronic phase, due to hepatocirrhosis, BCS may cause life-threatening gastrointestinal bleeding, refractory ascites and liver cancer (4). BCS is a type of congestive liver disease (5), which has a different pathogenesis, pathophysiological changes, clinical manifestations, and prognosis from those of common liver injuries caused by alcohol abuse, poisoning and viruses. As the worldwide incidence rate of BCS is only 1 in a million (1), research focusing on its pathogenesis is limited.

The essence of liver injury in BCS is passive hepatic congestion caused by hepatic venous outflow obstruction (HVCC) (5). In theory, hepatic congestion and hypoxia induce the pathological damage typical of BCS; however, there is a notable lack of empirical evidence in this field. However, studies on other types of HVCC suggest that liver hypoxia is associated with liver injury (5). It has been reported that congestive heart failure leads to passive hepatic congestion and forms a link between abnormal liver function and cardiovascular disease (6-8). In the present study, it was hypothesized that BCS may occur due to similar mechanisms. Hypoxia-induced injury may be an important contributor to BCS due to factors including ischemia and hypoxia in the liver at early stages. Chronic hypoxia may result in the production of excessive reactive oxygen species (ROS) and consequently oxidative stress (OS) (9), which are known to have an important role in various types of liver 
injury (10-12). Therefore, OS may also be involved in the formation of BCS lesions.

The most recognized predicative markers for OS are malonaldehyde (MDA) and superoxide dismutase (SOD). MDA is a product of lipid peroxidation and the most representative indicator of OS in the body, while SOD is representative of the body's antioxidant system, and its function is to clear ROS. These two markers indirectly reflect the extent of OS in the body (13). Endotoxin (ET) is closely associated with OS. When hepatic congestion occurs, intestinal ET enters the portal vein ectopically $(14,15)$. Early studies have reported that ET participates in liver congestion injury $(16,17)$. According to the present hypothesis, OS also has an important role in BCS, but studies to evidence this are currently lacking. MDA and SOD reflect the level of OS after liver congestion in BCS. ET caused by liver congestion and intestinal congestion due to BCS may affect OS. The aim of the present study was to detect the intrahepatic and extrahepatic levels of these markers at multiple time-points after establishment of a BCM model in order to clarify their significance in BCS, which has not been reported previously. In the present study, the method by Akiyoshi and Terada (18) was used to partially narrow the inferior vena cava (IVC) to establish a BCS animal model in rats. By analyzing changes in the hypoxia-associated markers MDA, SOD and ET in the serum of BCS patients and in the serum and liver homogenates of experimental animals at multiple time-points, the present study endeavored to explore the possible correlations between their levels and predicating BCS.

\section{Materials and methods}

Human samples. A total of 30 patients with BCS who presented at Anhui Provincial Hospital (Hefei, China) between January and June 2017 were randomly selected (all were diagnosed as having BCS by magnetic resonance imaging and Doppler ultrasound and the duration of BCS-associated symptoms at admission exceeded three months) as the patient group (males/females, 17/13; age, $45.2 \pm 11.3$ years). Furthermore, 30 healthy volunteers who took part in health examinations at Anhui Provincial Hospital Medical Center (Hefei, China) served as the healthy control group (males/females, 6/4; age, $46.7 \pm 11.5$ years). None of the subjects had any history of alcoholism, exposure to poison, pulmonary heart disease, viral hepatitis, immune hepatitis or other associated diseases. Blood samples $(5 \mathrm{ml})$ from control healthy volunteers or BCS patients prior to treatment was collected from the elbow vein and centrifuged at $1,500 \mathrm{x} \mathrm{g}$ at $4^{\circ} \mathrm{C}$ for $15 \mathrm{~min}$ to obtain the serum, which was stored at $-80^{\circ} \mathrm{C}$. All samples were obtained with informed consent and the analyses were approved by the Ethics Review Committee at Anhui Provincial Hospital (Hefei, China).

Animal model establishment. A total of 108 healthy adult male Sprague Dawley (SD) rats (weight, 185-235 g; age, 5-6 weeks), were purchased from Anhui Provincial Hospital Experimental Animal Center. All rats were housed under pathogen-free conditions at room temperature $\left(15-25^{\circ} \mathrm{C}\right)$, a humidity of $50-60 \%$ with a 12 -h light/dark cycle, and were provided with free access to food and water. All rats were divided into three groups according to a random number table (Fig. 1). The present study was approved by the Committee on the Ethics of Animal Experiments of Anhui Provincial Hospital. To establish the BCS model, 48 SD rats in the experimental group were fasted for 1 day (water provided) prior to routine disinfection and anesthesia. An open incision was made from the median incision of the xiphoid to separate the sickle-shaped ligament of the liver, exposing the IVC posterior to the liver. A $3 \mathrm{~F}$ microcatheter was placed in close contact with the IVC and withdrawn after being tightly wrapped with a 0 -gauge wire. Subsequently, the abdomen was sutured layer by layer. Rats in the Sham group underwent the same procedure except for the IVC ligation. All experimental group and Sham rats were intramuscularly injected with penicillin (20 U/day) for five consecutive days. The control rats were housed under normal conditions and were not subjected to any procedures.

Imaging and sample collection. A total of 9 rats from the sham and experimental groups were euthanized at the following time-points: 1, 3, 6 and 12 weeks post-surgery. Control rats were euthanized together with the rats from the sham and experimental groups at 6 weeks. The rats in each group were examined by digital subtraction angiography (DSA) 1 day prior to being sacrificed, and the blood flow through the hepatic vein, portal vein and IVC was observed. Blood samples were collected from the eye socket vein and centrifuged at $1,500 \mathrm{xg}$ at $4^{\circ} \mathrm{C}$ for $15 \mathrm{~min}$ to obtain the serum. After the rats were sacrificed, the liver was quickly removed and rinsed in saline at $4^{\circ} \mathrm{C}$. After being dried with filter paper, $0.5 \mathrm{~g}$ of liver tissue from each rat was homogenized and centrifuged at $1,125 \mathrm{x}$ g for $10 \mathrm{~min}$ and the supernatant was collected for further analysis. Part of the liver tissue from each rat was fixed in $10 \%$ formalin and then embedded in paraffin. All samples were stored at $-80^{\circ} \mathrm{C}$ until further analysis.

Hematoxylin and eosin (H\&E) staining. Specimens were sliced into 5- $\mu \mathrm{m}$ thick sections, stained with H\&E and the pathological changes in the liver tissues were observed with a light microscope at a magnification of x400 $(11,14)$.

Measurement of hypoxia indicators. MDA (rat, cat. no. xy-E12532; human, cat. no. xy-060c), SOD (rat, cat. no. xy-E12533; human, cat. no. xy-E10003) and ET (rat, cat. no. xy-E12516; human, cat. no. xy-E10857) levels in serum and liver homogenate were detected by double antigen sandwich assays (Shanghai Xin Yu Biological Technology Co., Ltd., Shanghai, China) according to the manufacturers' protocols.

Statistical analysis. Values are expressed as the mean \pm standard deviation, and statistical analysis was performed using SPSS version 22.0 statistical software (IBM Corp., Armonk, NY, USA). The Kolmogorov-Smirnov test was used to evaluate data normality. For multiple-group comparisons, analysis of variance was performed, followed by Tukey's honest significant difference test. The Pearson correlation coefficient was calculated for determining the correlations among hypoxia indicators. $\mathrm{P}<0.05$ was considered to indicate a statistically significant difference. 
Table I. Comparison of MDA levels (nmol/1) in the experimental animals ( $\mathrm{n}=9$ per group) in liver homogenate.

\begin{tabular}{lccrc}
\hline Week & Sham group & $\begin{array}{c}\text { Experimental } \\
\text { group }\end{array}$ & F-value & P-value \\
\hline 1 & $6.49 \pm 0.39$ & $8.55 \pm 0.45^{\mathrm{c}, \mathrm{d}}$ & 109.158 & 0.001 \\
3 & $6.61 \pm 0.43$ & $8.94 \pm 0.61^{\mathrm{c}}$ & 88.601 & 0.001 \\
6 & $6.48 \pm 0.57$ & $9.65 \pm 0.77^{\mathrm{a}, \mathrm{b}}$ & 99.146 & 0.001 \\
12 & $6.55 \pm 0.48$ & $9.41 \pm 0.21^{\mathrm{a}}$ & 267.812 & 0.001 \\
F-value & 0.141 & 7.176 & & \\
P-value & 0.935 & 0.001 & & \\
\hline
\end{tabular}

In the liver homogenate, the experimental group and the sham operation group were compared in terms of overall MDA levels, and the difference was statistically significant $(\mathrm{F}=219.016, \mathrm{P}=0.001)$. ${ }^{\mathrm{a}} \mathrm{P}<0.05$ vs. 1 week experiment group; ${ }^{\mathrm{b}} \mathrm{P}<0.05$ vs. 3 weeks experiment group; ${ }^{c} \mathrm{P}<0.05$ vs. 6 weeks experiment group; ${ }^{\mathrm{d}} \mathrm{P}<0.05$ vs. 12 weeks experiment group. MDA, malondialdehyde.

Table II. Comparison of serum MDA levels (nmol/l) in the experimental animals ( $\mathrm{n}=9$ per group).

\begin{tabular}{lcccc}
\hline Week & Sham group & $\begin{array}{c}\text { Experimental } \\
\text { group }\end{array}$ & F-value & P-value \\
\hline 1 & $6.63 \pm 0.68$ & $8.44 \pm 0.67$ & 32.392 & 0.001 \\
3 & $6.69 \pm 0.38$ & $8.91 \pm 0.64$ & 79.224 & 0.001 \\
6 & $6.49 \pm 0.53$ & $9.06 \pm 0.82$ & 63.134 & 0.001 \\
12 & $6.57 \pm 0.41$ & $8.44 \pm 0.59$ & 61.039 & 0.001 \\
F-value & 0.257 & 1.965 & & \\
P-value & 0.856 & 0.139 & & \\
\hline
\end{tabular}

In the serum, the experimental group and the sham operation group were compared in terms of overall MDA levels, and the difference was statistically significant $(\mathrm{F}=43.878, \mathrm{P}=0.001)$. MDA, malondialdehyde.

Table III. Comparison of SOD (pg/ml) levels in the experimental animals ( $\mathrm{n}=9$ per group) in liver homogenate.

\begin{tabular}{lccrc}
\hline Week & Sham group & $\begin{array}{c}\text { Experimental } \\
\text { Group }\end{array}$ & F-value & P-value \\
\hline 1 & $58.03 \pm 2.94$ & $54.48 \pm 4.40^{\mathrm{c}, \mathrm{d}}$ & 4.047 & 0.061 \\
3 & $57.82 \pm 2.23$ & $51.68 \pm 5.86^{\mathrm{c}, \mathrm{d}}$ & 8.629 & 0.010 \\
6 & $57.83 \pm 3.86$ & $38.32 \pm 5.26^{\mathrm{a}, \mathrm{b}}$ & 80.465 & 0.001 \\
12 & $58.43 \pm 4.75$ & $42.79 \pm 6.23^{\mathrm{a}, \mathrm{b}}$ & 35.888 & 0.001 \\
F-value & 0.058 & 17.053 & & \\
P-value & 0.981 & 0.001 & & \\
\hline
\end{tabular}

In the liver homogenate, the experimental group and the sham operation group were compared in terms of overall SOD levels, and the difference was statistically significant $(\mathrm{F}=19.317, \mathrm{P}=0.001)$. ${ }^{a} \mathrm{P}<0.05$ vs. 1 week experiment group; ${ }^{\mathrm{b}} \mathrm{P}<0.05$ vs. 3 weeks experiment group; ${ }^{\mathrm{c}} \mathrm{P}<0.05$ vs. 6 weeks experiment group; ${ }^{\mathrm{d}} \mathrm{P}<0.05$ vs 12 weeks experiment group. SOD, superoxide dismutase.

\section{Results}

Levels of hypoxia indicators in human serum. Serum MDA and ET levels in the patient group were significantly higher than those in the healthy group (both $\mathrm{P}=0.001$ ), whereas SOD levels were significantly lower ( $\mathrm{P}=0.001$; Fig. 2 ).

Establishment of the animal model. Successful model establishment in the experimental group was confirmed by DSA, with narrowing of the IVC posterior to the liver, distal lumen expansion and collateral circulation observed at 3-12 weeks post-surgery (Fig. 3A). Livers from each subgroup exhibited different degrees of pathological injury with liver fibrosis observed at 12 weeks (Fig. 3B). No pathological injury was observed in the sham or control groups.

Hypoxia indicator levels in BSC rats. The levels of MDA and ET in rat liver homogenate and serum were lower, and the SOD was higher in the sham group compared with the experimental group at 1, 3, 6 and 12 weeks post-operatively (MDA: $\mathrm{P}<0.001$; ET: $\mathrm{P}<0.001$; SOD: $\mathrm{P}<0.001$; Fig. 4, Tables I-VI). The MDA, ET, and SOD levels in both the liver homogenate and serum in the control group (Tables I-VI) were not significantly different from those of the sham group at 1, 3, 6 and 12 weeks post-operatively (MDA: $\mathrm{P}=0.950,0.916$; $\mathrm{ET}$ : $\mathrm{P}=0.941,0.976$; SOD: $\mathrm{P}=0.994,0.994$, respectively).

Comparing MDA, ET and SOD levels in the experimental group to those of the sham group at the same time-points, the levels of MDA and ET in rat liver homogenate and serum were lower, and the SOD was higher in the sham group compared with the experimental group at 3,6 and 12 weeks (Tables I-VI).

In the serum, the overall differences in MDA levels in the experimental group at the different time-points were not statistically significant $(\mathrm{P}=0.139)$, but those determined in the liver homogenate were significant $(\mathrm{P}=0.001)$. The MDA levels in the 6 weeks subgroup were significantly higher than those detected in the 1 and 3 weeks subgroups $(\mathrm{P}=0.001$ and 0.010 , respectively; Tables I and II).

In the serum, the overall differences in SOD and ET levels in the 6 weeks subgroup was significantly lower and higher, respectively, compared with the 1 and 3 weeks subgroups (all $\mathrm{P}<0.05$; Tables III-VI). The liver homogenate results were the same as those in the serum.

Correlations between MDA, SOD and ET levels. The time-dependent curves of MDA levels in the liver homogenate and sera in the experimental group were similar to the profiles recorded for the ET levels, all increasing from 1 week post-operatively, reaching a peak by 6 weeks, with a slight decrease by 12 weeks. The levels of MDA and ET in the experimental group at 1,3,6 and 12 weeks were higher than those in sham group, with a more pronounced increase rate (expressed data in experimental group/sham group x100\% at each time-point) in the liver homogenate than in the serum (Fig. 5). The time-dependent curves of the SOD levels in serum and liver homogenate were similar, with a decrease at 1 week post-operatively, reaching a valley by 6 weeks, prior to slightly increasing by 12 weeks. The SOD levels in the experimental subgroups were all lower than those in the 


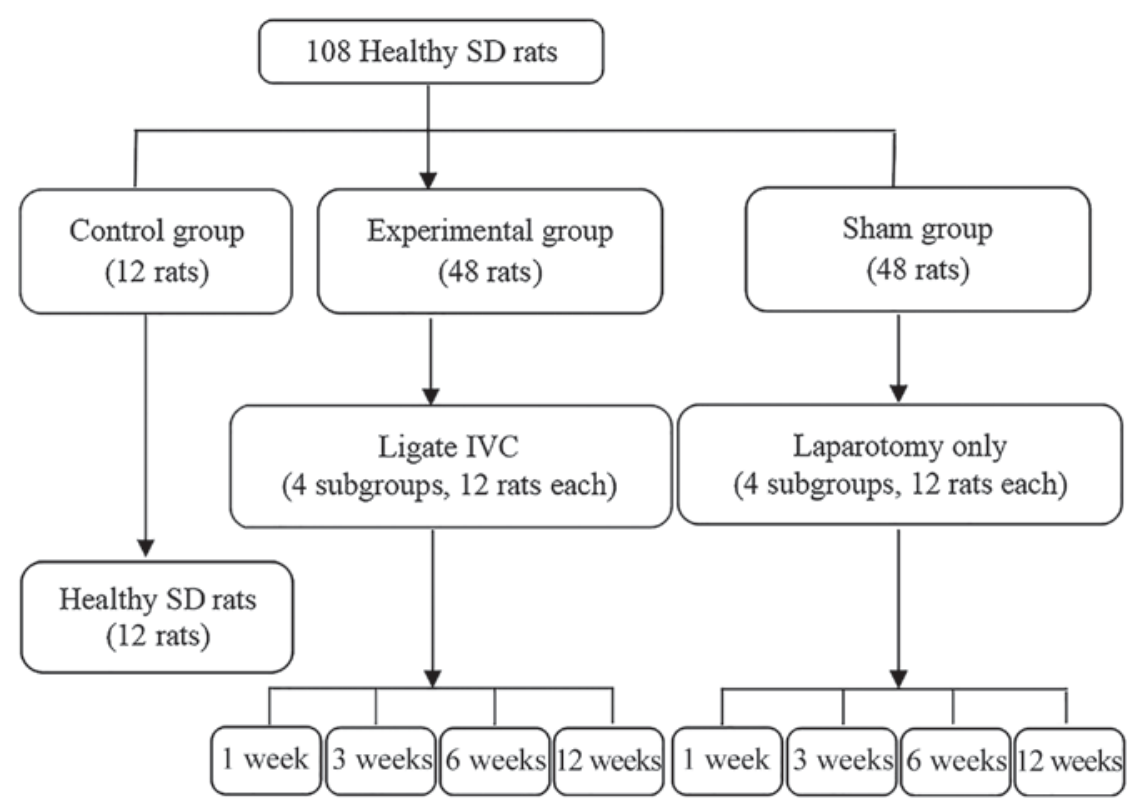

Figure 1. Flow chart illustrating the animal experiment and grouping. IVC, inferior vena cava; SD, Sprague Dawley.

sham group at each time-point, and the rate of the decline (expressed data in experimental group/sham group x100\% at each time-point) was higher in the liver homogenate than in the serum.

A negative correlation was determined between MDA and SOD in the liver homogenate and serum ( $\mathrm{r}=-0.814$ and $\mathrm{r}=-0.591$, respectively; both $\mathrm{P}=0.001$; Fig. 6 ), while a positive correlation was identified between MDA and ET ( $r=0.761$ and $r=0.422$; $\mathrm{P}=0.004$ and $\mathrm{P}=0.001$, respectively), and a negative correlation was determined between SOD and ET ( $r=-0.726$ and $r=-0.490$, respectively; both $\mathrm{P}=0.001$; Table VII and Fig. 6).

\section{Discussion}

The body uses oxygen as an electron acceptor in aerobic metabolism, producing ROS. ROS are closely associated with most metabolic, physiological and pathological processes in the body. When tissues are damaged by factors including ischemia and hypoxia, excessive ROS are produced, leading to OS (19). Electron transfer systems to produce ATP are the major source of ROS in the mitochondrial respiratory chain complex (20). The fact that the liver is rich in mitochondria makes it the major target organ for ROS-induced damage $(10,21)$. ROS have an important role in hepatic injury caused by congestion, hypoxia, virus infection, alcohol and poisoning (10-12). In early-stage BCS, disturbance of hepatic vein reflux may cause liver ischemia and hypoxia. Therefore, hypoxia may be the initiating factor of hepatic fibrosis and cirrhosis in BCS. In the present study, changes in indicators of hypoxia/OS were detected in BCS patients, and the results demonstrated that their levels were significantly different from those in healthy subjects, indicating that hypoxia/OS is associated with BCS. Furthermore, an animal model was established to study variations of these hypoxia-associated indicators in the progression of BCS.

MDA is one of the most commonly used representative indicators of OS injury (13). It is able to activate Kupffer

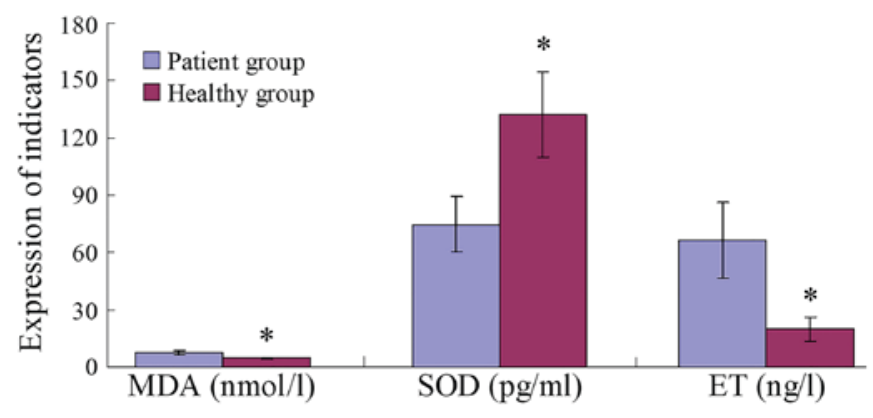

Figure 2. Hypoxia indicators in human patients. " $\mathrm{P}<0.001$ vs. Patient group. MDA, malondialdehyde; SOD, superoxide dismutase; ET, endotoxin.

cells to secrete numerous cytokines, activate hepatic stellate cells (HSC), and mediate HSC differentiation, proliferation and collagen synthesis (22). Another indicator of OS injury is SOD, which scavenges ROS, a representative of the body's antioxidant system (13). The increased/decreased levels of MDA/SOD in the experimental vs. those in the control group indicated that the hepatic vein occlusion induced intra- and extrahepatic lipid peroxidation injury. The increase in the levels of MDA and the decrease in the levels of SOD in liver homogenate were higher than those in the serum, which suggested that the liver in BCS had severe OS damage. This is mainly due to IVC obstruction of the hepatic vein outflow, leading to hepatic vein and IVC reflux disorders, which in turn causes obstacles in venous return to the liver in the lower extremities and various organs in the pelvic cavity. Due to the large volume directly located in the opening of the hepatic veins rich in mitochondria $(10,21)$, the liver suffers the heaviest degree of damage, which is consistent with the BCS-associated liver damage features that patients are clinically diagnosed with (1-3).

In the experimental group of the present study, MDA levels increased first and then decreased, which may be due to the direct oxidation of unsaturated fatty acids in the cell 

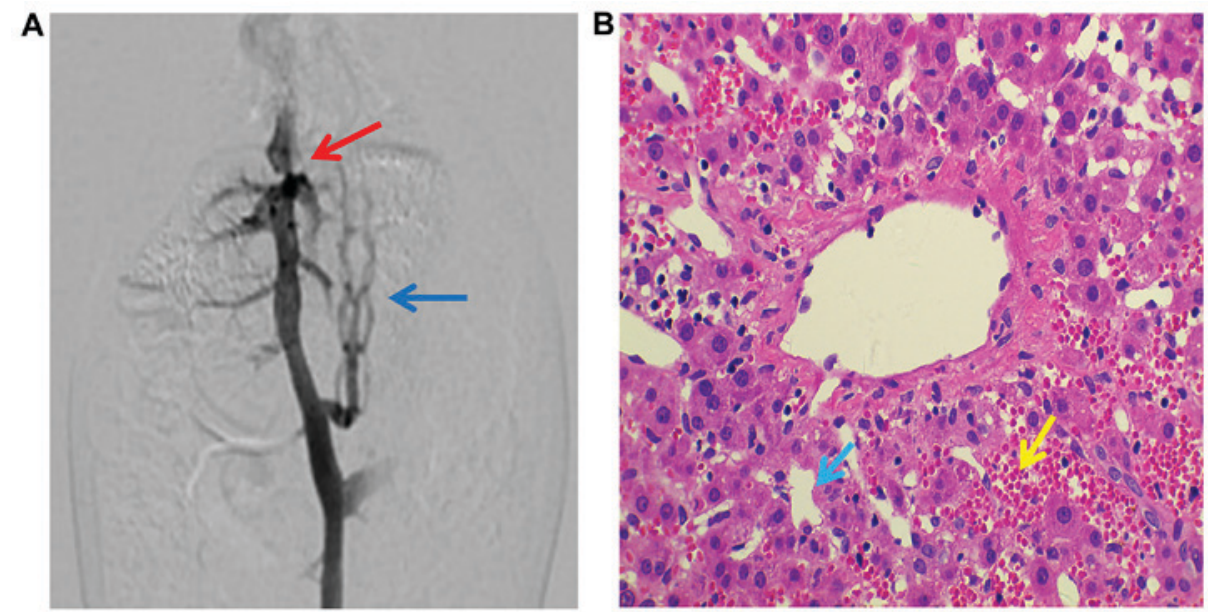

Figure 3. (A) Digital subtraction angiography indicated that the lumen of the inferior vena cava in the posterior segment of the liver from an experimental rat was significantly narrowed (red arrow) and the distal lumen was dilated with collateral circulation (blue arrow). (B) Hematoxylin and eosin staining revealed pathological changes in the liver from an experimental rat (magnification, $\mathrm{x} 400$ ): Abnormal arrangement of the hepatocyte cord, widened hepatic sinusoids (blue arrow) and a large number of red blood cells in the hepatic sinusoid (yellow arrow).
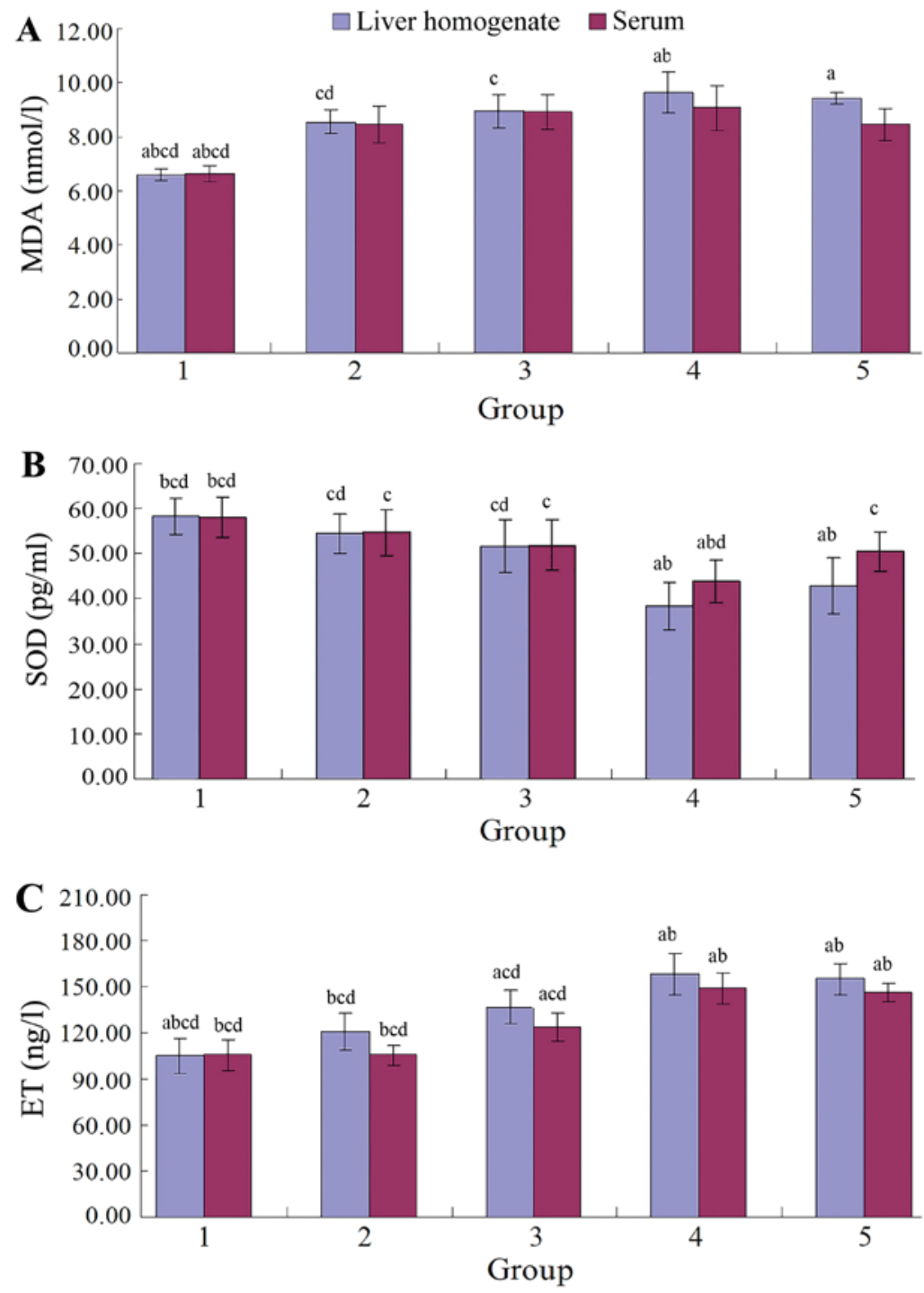

Figure 4. (A) MDA, (B) SOD and (C) ET levels in the liver homogenate and serum of the control and the experimental animal group at various time-points. MDA and ET increased post-operatively prior to reaching a peak by 6 weeks and decreasing slightly by 12 weeks. SOD decreased post-operatively prior to reaching a peak by 6 weeks and increasing slightly by 12 weeks. Groups: 1, control group; 2-5, experimental subgroup at 1, 3, 6 and 12 weeks, respectively. ${ }^{a} \mathrm{P}<0.05$ vs. 1 week experiment group; ${ }^{b} \mathrm{P}<0.05$ vs. 3 weeks experiment group; ${ }^{\mathrm{C}} \mathrm{P}<0.05$ vs. 6 weeks experiment group; ${ }^{\mathrm{d}} \mathrm{P}<0.05$ vs. 12 weeks experiment group; MDA, malondialdehyde; SOD, superoxide dismutase; ET, endotoxin. 

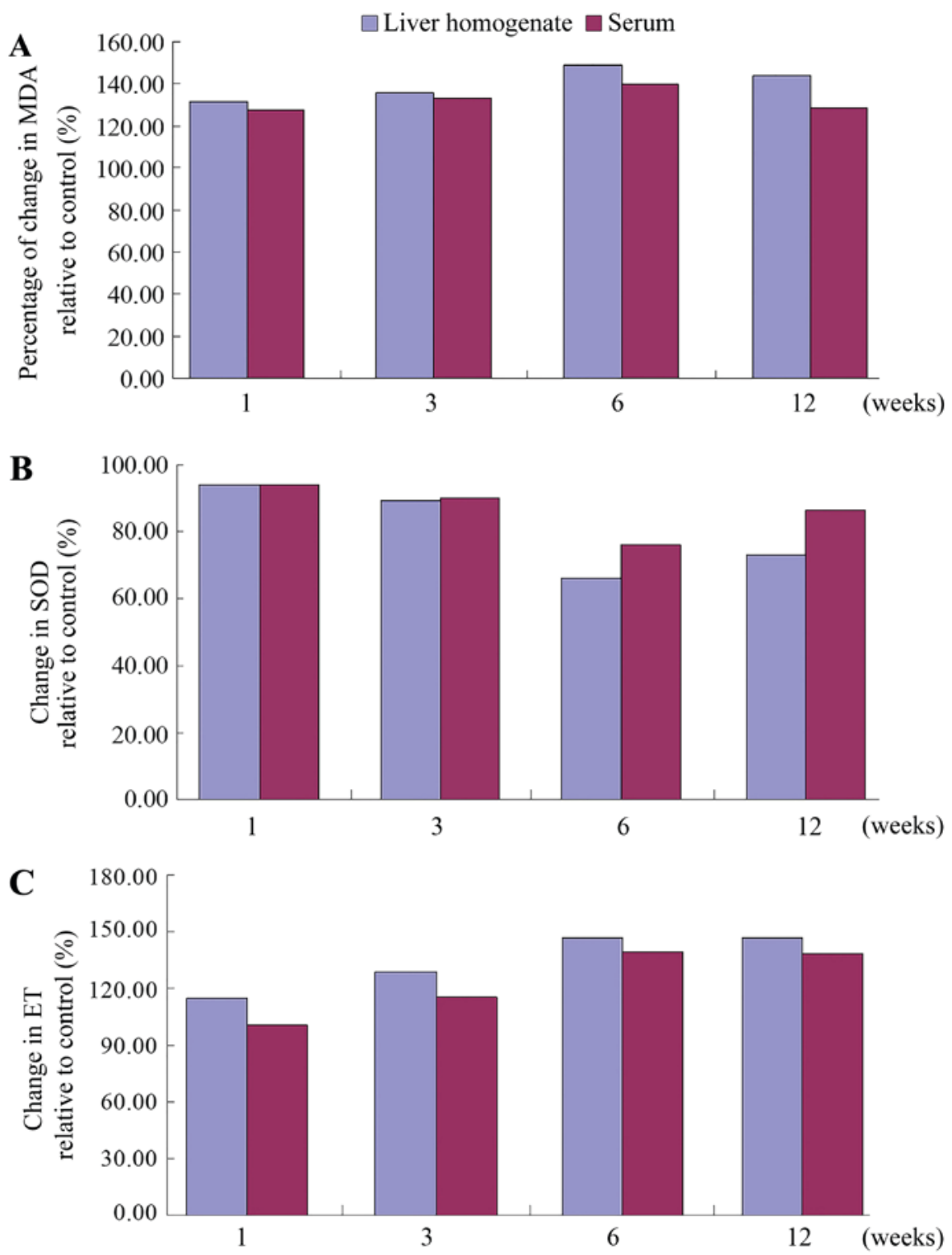

Figure 5. Changes in (A) MDA, (B) SOD and (C) ET levels over time in liver homogenate and serum. The changes in the levels were expressed as the experimental subgroup/sham subgroup x100\% at each time-point. MDA, malondialdehyde; SOD, superoxide dismutase; ET, endotoxin.

membrane to generate MDA during liver congestion and hypoxia, changing the fluidity and permeability of the cell membrane and leading to acute injury (23). Due to the innate antioxidant system, OS injury was not severe within the first three weeks of surgery. After three weeks, as ROS directly oxidize proteins, DNA and biological macromolecules, enzymes and non-enzymatic antioxidants were consumed in large quantities, aggravating the OS injury (24), resulting in a hypoxic injury peak by 6 weeks. Thereafter, the levels of oxidative stress declined, which may have been due to collateral circulation and compensatory blood supply alleviating the damage caused by ROS and the pressure of the portal vein. However, the level of MDA was still significantly higher than that of the control and sham groups, and the pathological liver damage was aggravated, indicating that collateral circulation compensation only temporarily alleviated OS damage, it did not fundamentally solve the BCS congestion and hypoxia-associated injury (25). Therefore, only the removal of the IVC obstruction was effective to treat the injury caused by congestion, which also confirmed the necessity for IVC angioplasty re-canalization in the clinical treatment of BCS $(25,26)$.

In the present study, intra- and extrahepatic ET levels in the experimental groups were higher than normal. The levels of ET in the liver homogenate were higher than those in the serum. This may be due to IVC obstruction directly leading to an increased pressure of the portal vein, resulting in gastrointestinal vein obstruction of blood backflow. In turn, this results in severe intestinal capillary blood flow stasis, rendering the intestine ischemic and hypoxic, leading to intestinal mucosal barrier dysfunction, followed by the translocation of intestinal bacteria and endotoxins $(14,15)$. ET entering the portal vein from the intestine is detoxified primarily by liver Kupffer cells, making the liver the predominant organ for ET aggregation (27). At one week post-operatively, no significant change in ET levels was noted in the experimental group. The reason for this may be that within the first week post-operatively, ET is confined to 
Table IV. Comparison of SOD $(\mathrm{pg} / \mathrm{ml})$ serum levels in the experimental animals ( $\mathrm{n}=9$ per group).

\begin{tabular}{lcccc}
\hline Weeks & Sham group & $\begin{array}{c}\text { Experimental } \\
\text { group }\end{array}$ & F-value & P-value \\
\hline 1 & $58.08 \pm 3.47$ & $54.69 \pm 5.03^{\mathrm{c}}$ & 2.768 & 0.116 \\
3 & $57.78 \pm 2.72$ & $51.93 \pm 5.54^{\mathrm{c}}$ & 8.094 & 0.012 \\
6 & $57.55 \pm 3.96$ & $43.75 \pm 4.75^{\mathrm{a}, \mathrm{b}, \mathrm{d}}$ & 44.763 & 0.001 \\
12 & $58.29 \pm 3.69$ & $50.45 \pm 4.44^{\mathrm{c}}$ & 16.651 & 0.001 \\
F-value & 0.080 & 7.903 & & \\
P-value & 0.971 & 0.001 & & \\
\hline
\end{tabular}

In the serum, the experimental group and the sham operation group were compared in terms of overall SOD levels, and the difference was statistically significant $(\mathrm{F}=31.643, \mathrm{P}=0.001)$. ${ }^{\mathrm{a}} \mathrm{P}<0.05$ vs. 1 week experiment group; ${ }^{\mathrm{b}} \mathrm{P}<0.05$ vs. 3 weeks experiment group; ${ }^{\mathrm{c}} \mathrm{P}<0.05$ vs. 6 weeks experiment group; ${ }^{\mathrm{d}} \mathrm{P}<0.05$ vs. 12 weeks experiment group. SOD, superoxide dismutase.

the portal vein and still in the clearance capacity range of the hepatic Kupffer cells, so that ET levels in the serum do not significantly increase in the short-term. However, the ET rapidly increased thereafter, which may be due to persistent hypoxia and portal hypertension secondary to IVC obstruction, causing the liver to gather excessive ET $(14,15,27)$, beyond the influence of detoxifying Kupffer cells after entering the systemic circulation. The level of ET decreased slightly after 6 weeks, indicating that the initiation of the collateral circulation temporarily relieved endotoxemia.

In the present study, the ET levels in the liver homogenate and serum of BCS rats was positively correlated with MDA levels and negatively correlated with SOD levels, suggesting that ET may damage the liver through OS, which is supported by the results of studies on other types of liver injury (16). This is due to ligation of the IVC posterior to the liver resulting in an obstruction of outflow from the hepatic veins and IVC, leading to hepatic congestion and hypoxia, resulting in the production of a large number of ROS, which may activate hypoxia-inducible factor (HIF)-1, nuclear factor (NF)- $\kappa B$, and various cytokines, consequently activating HSC to cause hepatic fibrosis (22). Furthermore, after ligation of the IVC, the portal pressure continues to rise and large amounts of ET enter the blood system. The ET degradation product lipid A inhibits ATP synthesis, affecting mitochondrial energy synthesis and respiratory chain electron transport, and inducing the generation of ROS, which in turn aggravates liver injury (14). In addition, ET enhances the expression of CD14 and Toll-like receptor 4 in Kupffer cells, inducing the production of a large number of cytokines, including tumor necrosis factor- $\alpha$. This may increase the inflammatory reaction (17), which increases intrahepatic resistance and splanchnic blood flow, leading to further increases in venous pressure, hepatic venous congestion and hypoxia (15). These results demonstrate that after IVC ligation posterior to the liver, a self-aggravating cycle of congestion, hypoxia-induced ROS and rising ET levels was generated. This may be the mechanism of the sustained liver injury observed in BCS
Table V. Comparison of ET (ng/l) levels in liver homogenate in the experimental animals ( $\mathrm{n}=9$ per group).

\begin{tabular}{lccrc}
\hline Week & Sham group & $\begin{array}{c}\text { Experimental } \\
\text { group }\end{array}$ & F-value & P-value \\
\hline 1 & $104.93 \pm 5.89$ & $120.66 \pm 11.97^{\mathrm{b}, \mathrm{c}, \mathrm{d}}$ & 12.498 & 0.003 \\
3 & $106.23 \pm 8.97$ & $136.69 \pm 10.91^{\mathrm{a}, \mathrm{d}, \mathrm{d}}$ & 41.844 & 0.000 \\
6 & $107.87 \pm 7.62$ & $158.21 \pm 13.44^{\mathrm{a}, \mathrm{b}}$ & 95.519 & 0.000 \\
12 & $105.72 \pm 4.94$ & $154.94 \pm 10.38^{\mathrm{a}, \mathrm{b}}$ & 165.022 & 0.000 \\
F-value & 0.281 & 19.870 & & \\
P-value & 0.838 & 0.001 & & \\
\hline
\end{tabular}

In the liver homogenate, the experimental group and the sham operation group were compared in terms of overall ET levels, and the difference was statistically significant $(\mathrm{F}=105.515, \mathrm{P}=0.001)$. ${ }^{\mathrm{a}} \mathrm{P}<0.05$ vs. 1 week experiment group; ${ }^{\mathrm{b}} \mathrm{P}<0.05$ vs. 3 weeks experiment group; ${ }^{\mathrm{C}} \mathrm{P}<0.05$ vs. 6 weeks experiment group; ${ }^{\mathrm{d}} \mathrm{P}<0.05$ vs. 12 weeks experiment group. ET, endotoxin.

Table VI. Comparison of ET (ng/l) levels in serum in the experimental animals ( $\mathrm{n}=9$ per group).

\begin{tabular}{lccrc}
\hline Week & Sham group & $\begin{array}{c}\text { Experimental } \\
\text { group }\end{array}$ & F-value & P-value \\
\hline 1 & $104.87 \pm 6.54$ & $105.56 \pm 7.77^{\mathrm{b}, \mathrm{c}, \mathrm{d}}$ & 0.041 & 0.842 \\
3 & $107.31 \pm 9.06$ & $123.67 \pm 12.77^{\mathrm{a}, \mathrm{d}, \mathrm{d}}$ & 9.832 & 0.006 \\
6 & $106.72 \pm 10.23$ & $148.97 \pm 8.46^{\mathrm{a}, \mathrm{b}}$ & 91.193 & 0.000 \\
12 & $105.66 \pm 6.02$ & $145.91 \pm 9.21^{\mathrm{a}, \mathrm{b}}$ & 120.323 & 0.000 \\
F-value & 0.160 & 39.372 & & \\
P-value & 0.923 & 0.001 & & \\
\hline
\end{tabular}

In the serum, the experimental group and the sham operation group were compared in terms of overall ET levels, and the difference was statistically significant $(\mathrm{F}=46.323, \mathrm{P}=0.001)$. ${ }^{\mathrm{a}} \mathrm{P}<0.05$ vs. 1 week experiment group; ${ }^{\mathrm{b}} \mathrm{P}<0.05$ vs. 3 weeks experiment group; ${ }^{\mathrm{c}} \mathrm{P}<0.05$ vs. 6 weeks experiment group; ${ }^{\mathrm{d}} \mathrm{P}<0.05$ vs. 12 weeks experiment group. ET, endotoxin.

patients, and may explain why BCS patients have a low clinical self-healing capacity.

The results of the present study indicate that IVC obstruction in BSC rats increases hypoxia-associated ROS and ET levels, leading to OS and endotoxemia, which may be the dominant causes of BCS-associated liver injury. However, how hypoxia induces the activation of nuclear transcription factors (HIF-1 $\alpha$, HIF-2 $\alpha$ and NF- $\mathrm{kB}$ ) and regulates the expression of downstream target genes, which in turn influence the integrity of the extracellular matrix, angiogenesis, growth factors and their receptors, and the molecular mechanisms that eventually lead to congestive cirrhosis, remain to be fully elucidated and should be further investigated.

In conclusion, in the early stages of BCS, reflux disorders of the hepatic vein and IVC cause congestion and hypoxia, particularly in the liver. With disease progression, congestion and hypoxia tended to worsen, but were slightly relieved with the emergence of collateral circulation during the later 
Table VII. Correlation analysis of hypoxia indicators.

\begin{tabular}{lllr}
\hline Correlation & MDA and SOD & MDA and ET & SOD and ET \\
\hline Serum & $\mathrm{R}=-0.591, \mathrm{P}=0.001$ & $\mathrm{R}=0.422, \mathrm{P}=0.004$ & $\mathrm{R}=-0.490, \mathrm{P}=0.001$ \\
Liver homogenate & $\mathrm{R}=-0.814, \mathrm{P}=0.001$ & $\mathrm{R}=0.761, \mathrm{P}=0.001$ & $\mathrm{R}=-0.726, \mathrm{P}=0.001$
\end{tabular}

MDA, malondialdehyde; SOD, superoxide dismutase; ET, endotoxin.
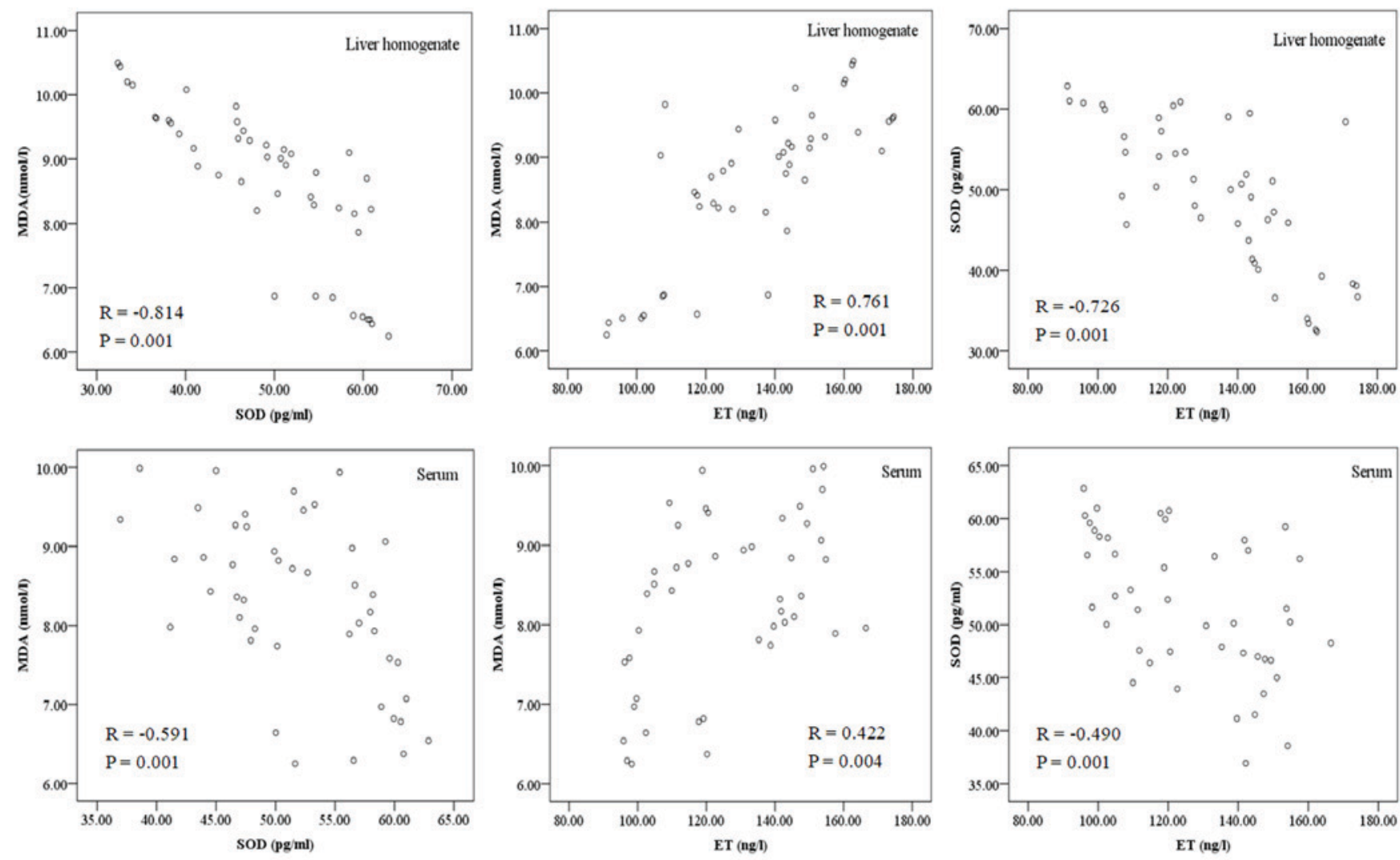

Figure 6. Correlations between MDA, SOD and ET levels in liver homogenate and serum. MDA, malondialdehyde; SOD, superoxide dismutase; ET, endotoxin.

stages, while they still remained at levels that were higher than normal. Thus, hypoxia is a common factor that is involved during all stages of BCS, and may be the principal factor in the initiation and aggravation of BCS.

\section{Acknowledgements}

Not applicable.

\section{Funding}

This project was supported by Natural Science Foundation of Anhui Province (grant no. 1708085QH218).

\section{Availability of data and materials}

The datasets used and/or analyzed during the current study are available from the corresponding author on reasonable request.

\section{Authors' contributions}

D-LC, NZ and C-TL designed the study. D-LC, W-WF, C-LL performed the experiments. D-LC, NZ and YL collected and analyzed the data. W-WF, NZ and C-LL contributed to sample collection and inputted the data into the computer. D-LC and NZ drafted and wrote the manuscript. W-FL, C-LL and C-TL gave advice on the experimental design, interpreted the results and critically revised the manuscript. All authors read and approved the final version of the manuscript.

\section{Ethics approval and consent to participate}

The present study was approved by the Ethics Review Committee at Anhui Provincial Hospital. All procedures were performed in accordance with the ethical standards of the responsible committee on human experimentation (institutional and national) and with the Helsinki Declaration of 
1975, as revised in 2008 (5). Informed consent was obtained from all patients for being included in the study.

\section{Patient consent for publication}

Not applicable.

\section{Competing interests}

The authors declare that they have no competing interests.

\section{References}

1. Martens P and Nevens F: Budd-Chiari syndrome. United European Gastroenterol J 3: 489-500, 2015.

2. Aydinli M and Bayraktar Y: Budd-Chiari syndrome: Etiology, pathogenesis and diagnosis. World J Gastroenterol 13: 2693-2696, 2007.

3. Pavri TM, Herbst A, Reddy R and Forde KA: Budd-Chiari syndrome: A single-center experience. World J Gastroenterol 20 : 16236-16244, 2014.

4. Akamatsu N, Sugawara Y and Kokudo N: Budd-Chiari syndrome and liver transplantation. Intractable Rare Dis Res 4: 24-32, 2015.

5. Bayraktar UD, Seren S and Bayraktar Y: Hepatic venous outflow obstruction: Three similar syndromes. World J Gastroenterol 13: 1912-1927, 2007.

6. Maleki M, Vakilian F and Amin A: Liver diseases in heart failure. Heart Asia 3: 143-149, 2011.

7. Fouad YM and Yehia R: Hepato-cardiac disorders. World J Hepatol 6: 41-54, 2014.

8. Waseem $\mathrm{N}$ and Chen PH: Hypoxic hepatitis: A review and clinical update. J Clin Transl Hepatol 4: 263-268, 2016.

9. Debevec T, Millet GP and Pialoux V: Hypoxia-induced oxidative stress modulation with physical activity. Front Physiol 8: 84, 2017.

10. Li S, Tan HY, Wang N, Zhang ZJ, Lao L, Wong CW and Feng Y: The role of oxidative stress and antioxidants in liver diseases. In J Mol Sci 16: 26087-26124, 2015.

11. Feng Y, Wang N, Ye X, Li H, Feng Y, Cheung F and Nagamatsu T: Hepatoprotective effect and its possible mechanism of Coptidis rhizoma aqueous extract on carbon tetrachloride-induced chronic liver hepatotoxicity in rats. J Ethnopharmacol 138: 683-690, 2011.

12. Singal AK, Jampana SC and Weinman SA: Antioxidants as therapeutic agents for liver disease. Liver Int 31: 1432-1448, 2011.

13. Bajpai J, Prakash V, Kant S, Verma AK, Srivastava A, Bajaj DK, Ahmad MK and Agarwal A: Study of oxidative stress biomarkers in chronic obstructive pulmonary disease and their correlation with disease severity in north Indian population cohort. Lung India 34: 324-329, 2017.

14. Xu H, Xiong J, Xu J, Li S, Zhou Y, Chen D, Cai X, Ping J, Deng $M$ and Chen J: Mosapride stabilizes intestinal microbiota to reduce bacterial translocation and endotoxemia in $\mathrm{CCl}_{4}$-induced cirrhotic rats. Dig Dis Sci 62: 2801-2811, 2017.
15. Zhao TY, Su LP, Ma CY, Zhai XH, Duan ZJ, Zhu Y, Zhao G, Li CY, Wang LX and Yang D: IGF-1 decreases portal vein endotoxin via regulating intestinal tight junctions and plays a role in attenuating portal hypertension of cirrhotic rats. BMC Gastroenterol 15: 77, 2015.

16. Abdel-Salam OME, Morsy SMY and Sleem AA: The effect of different antidepressant drugs on oxidative stress after lipopolysaccharide administration in mice. EXCLI J 10: 290-302, 2011.

17. Miao CM, He K, Li PZ, Liu ZJ, Zhu XW, Ou ZB, Ruan XZ, Gong JP and Liu CA: LXR $\alpha$ represses LPS-induced inflammatory responses by competing with IRF3 for GRIP1 in Kupffer cells. Int Immunopharmacol 35: 272-279, 2016.

18. Akiyoshi $\mathrm{H}$ and Terada $\mathrm{T}$ : Centrilobular and perisinusoidal fibrosis in experimental congestive liver in the rat. J Hepatol 30: 433-43, 1999.

19. Kuksal N, Chalker J and Mailloux RJ: Progress in understanding the molecular oxygen paradox-function of mitochondrial reactive oxygen species in cell signaling. Biol Chem 398: 1209-1227, 2017.

20. Kadlec AO, Beyer AM, Ait-Aissa K and Gutterman DD: Mitochondrial signaling in the vascular endothelium: Beyond reactiveoxygen species. Basic Res Cardiol 111: 26, 2016.

21. Sánchez-Valle V, Chávez-Tapia NC, Uribe $M$ and Méndez-Sánchez N: Role of oxidative stress and molecular changes in liver fibrosis: A review. Curr Med Chem 19: 4850-4860, 2012.

22. Cong T, Jin XY, Zhao L, Ma L, Li RS, Zhao P and Guo CJ: Anti-fibrotic effects of the Masson pine pollen aqueous extract on hepatic fibrosis rat model. Int J Clin Exp Pathol 8: 4651-4661, 2015.

23. Sharma A, Sharma MK and Kumar M: Modulatory role of Emblica officinalis fruit extract against arsenic induced oxidative stress in Swiss albino mice. Chem Biol Interact 180: 20-30, 2009.

24. Anavi S, Ni Z, Tirosh O and Fedorova M: Steatosis-induced proteins adducts with lipid peroxidation products and nuclear electrophilic stress in hepatocytes. Redox Biol 4: 158-168, 2015.

25. Fu Y, Sun YL, Ma XX, Xu PQ, Feng LS, Tang Z, Guan S, Wang ZW and Luo CH: Necessity and indications of invasive treatment for Budd-Chiari syndrome. Hepatobiliary Pancreat Dis Int 10: 254-60, 2011.

26. Qi XS, Ren WR, Fan DM and Han GH: Selection of treatment modalities for Budd-Chiari Syndrome in China: A preliminary survey of published literature. World J Gastroenterology 20: 10628-10636, 2014.

27. Thomas P, Hayashi H, Lazure D, Burke PA, Bajenova O, Ganguly A and Forse RA: Inhibition of LP-S activation of Kupffer cells by transition metals. J Surg Res 148: 116-120, 2008.

(i) $($ This work is licensed under a Creative Commons Attribution-NonCommercial-NoDerivatives 4.0 International (CC BY-NC-ND 4.0) License. 